\title{
Tipos de fruto de Yucca filifera y formas asociadas de Tegeticula yuccasella (Lepidoptera)
}

Las especies del género $Y u c c a$ (Agavaceae) y las del género Tegeticula (Lepidoptera: Incurvariidae) mantienen una relación mutualista (Aker y Udovic, 1981; Powell, 1992), la cual en términos generales se presenta de la siguiente manera. Los adultos de Tegeticula emergen del suelo durante la época de floración de estas plantas. Las hembras recogen polen antes o después del apareamiento. Seleccionan flores recién abiertas, ovipositan en el interior de los pistilos e inmediatamente después polinizan a la misma flor. Los frutos inician su desarrollo y en su interior los huevecillos eclosionan, las larvas que emergen se alimentan de las semillas, después salen de los frutos antes de que éstos maduren, descienden al suelo y se entierran, forman un capullo e hibernan. Al año siguiente, unos días antes de que inicie la floración de las yucas, se forma la pupa y luego los adultos emergen para reiniciar el ciclo (Riley, 1892; Trelease, 1893; Rau, 1945; Powell y Mackie, 1966; Aker y Udovic, 1981).

Para efectuar la oviposición las hembras de Tegeticula penetran la pared del pistilo con el ovipositor. La punción del ovario con el tiempo da lugar a que se forme una depresión en el fruto, lo cual se debe a que en el área circundante se inhibe el desarrollo de los óvulos. El conjunto de punciones en el ovario origina la deformación de los frutos, característica de este género de plantas (Riley, 1892; Davis, 1967). En Yucca se observan diferentes tipos de fruto según la deformación que presentan; existen frutos con curvatura, con constricción media y sin deformación aparente. Por ejemplo, en $Y$. schottii los frutos presentan una curvatura pronunciada, esto se debe a que $T$. yuccasella oviposita hacia la parte media del pistilo en un sólo lóculo (Davis, 1967). Y. filamentosa es una de las especies que produce frutos con constricción media debido a que el polinizador también oviposita hacia la mitad del pistilo, pero lo hace en cada uno de los lóculos (Riley, 1892), es evidente que la constricción resulta de la inhibición del desarrollo de los óvulos en los sitios de oviposición distribuidos alrededor del pistilo (Davis, 1967). Los frutos sin deformación aparente se presentan en especies como $Y$. brevifolia en donde el polinizador, $T$. synthetica, no oviposita en el ovario, lo hace en el estilo, por lo que los óvulos no son afectados (Trelease, 1893; Davis, 1967) y los frutos no sufren deformación.

En cuanto a Y. filifera, en recorridos de campo realizados en el estado de Hidalgo, observamos que en algunas localidades la planta produce frutos con curvatura, los cuales ya habían sido reportados para esta especie (Davis, 1967), y en otras, frutos con constricción distal, que no estaban descritos.
Considerando que el tipo de deformación de los frutos de Yucca depende del sitio en el pistilo en el que Tegeticula oviposite, era obvio suponer que en $Y$. filifera el polinizador T. yuccasella, presenta un comportamiento variado al ovipositar; en el caso de los frutos con curvatura oviposita hacia la parte media del pistilo y en los frutos con constricción distal lo hace hacia el tercio superior. En este punto se cuestionó si además de las supuestas diferencias comportamentales, habría otras características diferenciales entre $T$. yuccasella asociada a cada tipo de fruto. Estas observaciones sirvieron como base para plantear el presente estudio que tuvo como objetivo determinar cuántos tipos de fruto produce $Y$. filifera y verificar si $T$. yuccasella asociada a cada uno de ellos es diferente.

Para determinar los tipos de frutos que produce $Y$. filifera, se visitaron distintos puntos en el área de distribución de esta especie de julio a octubre de 1988 y 1989, y en cada uno de ellos se hicieron observaciones directas y se tomaron muestras para herbario las cuales están depositadas en el Centro de Investigaciones Biológicas de la Universidad Autónoma del Estado de Hidalgo.

Para establecer si T. yuccasella asociada a cada tipo de fruto de $Y$. filifera es diferente, se compararon las dimensiones de la genitalia de los adultos de este insecto obtenidos a partir de larvas emergidas de cada tipo de fruto. Este método se eligió porque Davis (1967) sugirió que algunos caracteres de la genitalia podrían tener significado taxonómico en este grupo de insectos, en especial la longitud de la genitalia de los machos. Los adultos se obtuvieron como se describe a continuación. Se colectaron frutos, antes de su maduración, en dos localidades del estado de Hidalgo; uno de los tipos se colectó en la colonia 20 de Noviembre, municipio de El Arenal, en el Valle del Mezquital, y el otro en el ejido Villa Margarita, municipio de Zempoala, en el Valle de México. En el laboratorio los frutos se colocaron en bolsas de plástico, separados por localidad, y se revisaron diariamente para capturar a las larvas que iban saliendo, éstas fueron colocadas en frascos de vidrio de 3 litros que contenían $1.5 \mathrm{~kg}$ de suelo previamente esterilizado proveniente del mismo sitio de colecta de los frutos. Al año siguiente, a partir de marzo, los frascos se revisaron periódicamente para capturar a los adultos de T. yuccasella que emergían del suelo. De estos adultos se obtuvo la genitalia que fue medida con un ocular micrométrico.

Se encontró que $Y$. filifera produce dos tipos de fruto, según la deformación que presentan: con curvatura y con constricción distal (Fig. 1). Se observó que dentro de cada tipo existe variabilidad, tanto de la curvatura como de la 


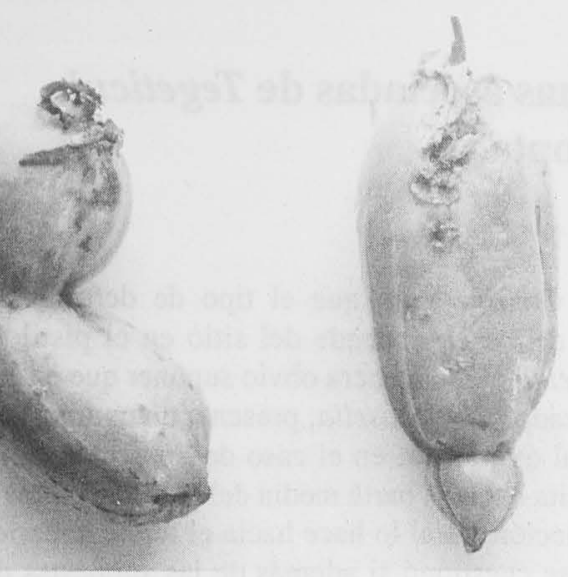

Figura. 1. Tipos de fruto producidos por Yucca filifera. A la izquierda fruto con curvatura, a la derecha fruto con constricción distal, éste último mide $7.7 \mathrm{~cm}$ de largo.

constricción distal, las cuales pueden estar menos pronunciadas que las de los frutos que se presentan en la Fig. 1, pero no se determinó el porcentaje de variabilidad. Sólo en una localidad, en el municipio de Santiago de Anaya, Hidalgo, ubicada en el Valle del Mezquital se registraron algunos ejemplares de $Y$. filifera que producen frutos con deformación indefinida, tal que no pudieron clasificarse en ninguno de los dos tipos mencionados.

Se determinó que en Hidalgo las poblaciones de $Y$. filifera que crecen en el Valle de México sólo producen frutos con constricción distal, mientras que en el Valle del Mezquital solamente producen frutos con curvatura, a excepción de algunas plantas encontradas en Santiago de Anaya que presentan frutos indefinidos. En la Barranca de Metztitlán también se producen frutos con curvatura. En los estados de Querétaro, Guanajuato, San Luis Potosí, Nuevo León y Tamaulipas se observaron tanto frutos con curvatura como frutos con constricción distal. En estas entidades ambos tipos de fruto se encontraron inclusive en una misma localidad, pero no se determinó su abundancia, tampoco se definió si un mismo individuo produce uno o dos tipos de frutos en la misma temporada y en temporadas subsecuentes.

Con base en observaciones realizadas en los frutos de $Y$. filifera en cuanto a su deformación y a la ubicación de las depresiones, que señalan los sitios en los que se efectuó la punción del pistilo, se pudo inferir que el comportamiento de oviposición difiere entre las poblaciones de T. yuccasella que intervienen en la formación de frutos con curvatura y las que intervienen en la formación de frutos con constricción distal. En el primer caso, T. yuccasella oviposita hacia la parte media del pistilo en un sólo lóculo y en el segundo lo hace en el tercio superior depositando los huevecillos en cada lóculo. Otros autores han reportado casos en los cuales T. yuccasella se comporta de manera diferente al ovipositar. Riley (1892) señaló que en $Y$. filamentosa, $T$. yuccasella usualmente se coloca con la cabeza dirigida hacia el estigma y que en esa posición oviposita en la parte media del estilo, pero que no era infrecuente observarla ovipositando con la cabeza dirigida hacia la base del ovario, en cuyo caso los frutos mostraban las punciones hacia la parte superior. Aparentemente Riley no prestó mayor atención a sus observaciones. Powell (1992) menciona que Addicott, cuyos trabajos no pudieron ser consultados, sospechaba que en ciertas yucas, Tegeticula presenta especies hermanas simpátricas con formas que ovipositan superficial o profundamente.

En cuanto a la obtención de adultos de T. yuccasella, de 35 frutos con curvatura colectados en 1989 en la colonia 20 de Noviembre se recuperaron 40 larvas y al año siguiente emergieron 17 adultos. Mientras que de 25 frutos con constricción distal colectados en 1989 en el ejido Villa Margarita, surgieron 29 larvas y emergieron 16 adultos. De algunos de estos se extrajo la genitalia y en la de las hembras se midió el diámetro del signum y largo de la bursa y en la de los machos el largo total. El largo de la genitalia de los machos se determinó midiendo la longitud existente entre el ápice distal del sacus y el extremo terminal de las valvas. En el Cuadro 1 se presentan las medidas obtenidas.

En los datos del Cuadro 1 se observa que las dimensiones de la genitalia de hembras y machos de $T$. yuccasella asociados a frutos con curvatura son mayores que la de los insectos asociados a frutos con constricción distal. La mag-

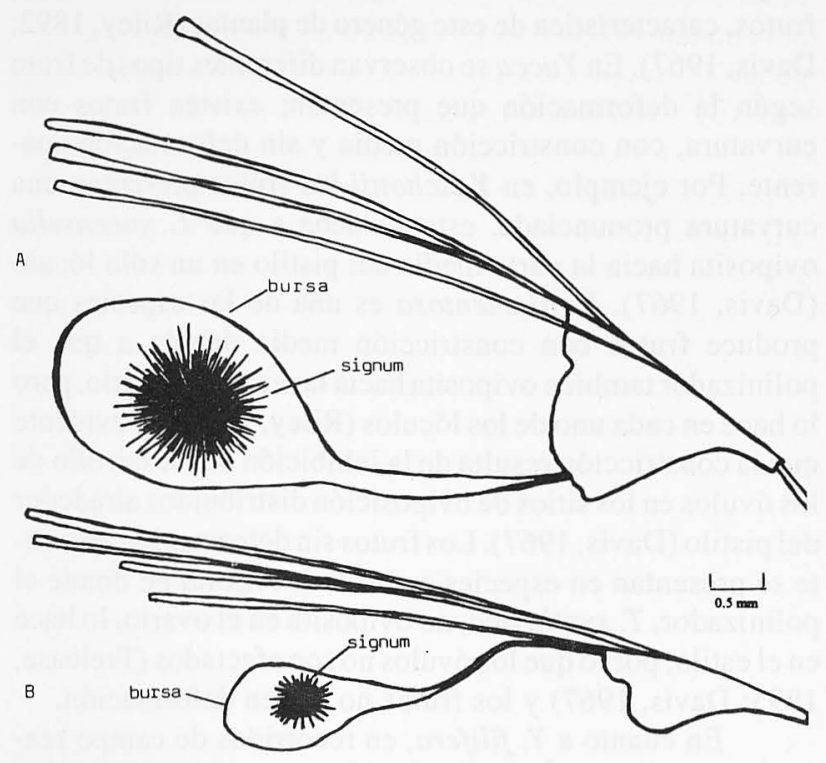

Figura. 2. Genitalia de la hembra de Tegeticula yuccasella asociada a frutos con curvatura (A) y a frutos con constricción distal (B) producidos por Yucca filifera. 
CuADro 1. Medidas promedio e intervalo en mm, de la genitalia de Tegeticula yucasella asociada a cada tipo de fruto de Yucca filifera.

\begin{tabular}{|c|c|c|c|}
\hline & \multicolumn{2}{|c|}{ hembra $^{1}$} & macho ${ }^{2}$ \\
\hline Fruto con: & diámetro signum & largo bursa & largo genitalia \\
\hline Curvatura & $1.40(1.39-1.41)$ & $2.25(2.24-2.26)$ & $2.80(2.80-2.81)$ \\
\hline $\begin{array}{l}\text { Constricción } \\
\text { distal }\end{array}$ & $0.77(0.77-0.78$ & $1.54(1.53-1.56)$ & $2.45(2.44-2.46)$ \\
\hline
\end{tabular}

${ }^{1} \mathrm{~N}=4$, por tipo de fruto, ${ }^{2} \mathrm{~N}=2$, por tipo de fruto

nitud de la diferencia en las dimensiones de la genitalia es mayor en las hembras. En la Fig. 2 se presentan dibujos de la genitalia de hembras de T. yuccasella asociadas a cada tipo de fruto en donde se aprecia la diferencia de tamaño entre cada una. Se consideró que la diferencia en las dimensiones de la genitalia representa una evidencia de que $T$. yuccasella asociada a cada tipo de fruto es diferente, que se trata de dos entidades biológicas distintas a las que en este estudio se les denominó formas. Sin embargo, es necesario confirmar su existencia realizando estudios con un número mayor de ejemplares y analizando los datos estadísticamente. Por lo pronto, las diferencias morfológicas en la genitalia y las supuestas diferencias comportamentales, apoyan la idea de que efectivamente $T$. yuccasella, el polinizador de $Y$. filifera, está constituído por más de una entidad biológica. Esto concuerda con lo que han señalado otros autores como Davis (1967), quien reconoció que el tamaño de la genitalia de los machos exhibe una variación inusual, por lo que sugirió que en $T$. yuccasella podrían estar presentes varias razas o especies hermanas. Por su parte, Powell (1992), con base en una serie de evidencias morfológicas, fenológicas y geográficas encontradas por él mismo y por otros autores como Davis (1967), indicóque las poblaciones de T. yuccasella asociadas a diferentes yucas en Nuevo México y Arizona son entidades biológicas separadas que están aisladas espacial y/ o temporalmente y por la especificidad de hospederos y que actúan como especies.

Con los resultados obtenidos en este estudio se alcanzó el objetivo planteado ya que se determinó el número de tipos de frutos que $Y$. filifera produce y se evidenció que a cada uno de ellos se encuentra asociada una forma distinta de T. yuccasella.

\section{LITERATURA CITADA}

Aker CL, Udovic D. 1981. Oviposition and pollination behavior of the yucca moth, Tegeticula maculata (Lepidoptera: Prodoxidae), and its relation to the reproductive biology of Yucca whipplei (Agavaceae). Oecologia 49: 96-101.

Davis DR. 1967. A revision of the moths of the subfamily Prodoxinae (Lepidoptera: Incurvariidae). United States National Museum Bulletin 255: 1-170.

Powell JA, Mackie RA. 1966. Biological interrelationships of moths and Yucca whipplei (Lepidoptera: Gelechiidae, Blastobasidae, Prodoxidae). Univeristy of California Publications in Entomology 42: 1-59.

Powell JA. 1992. Interrelationships of yuccas and yucca moths. Trends in Ecology and Evolution 7: 10-15.

Rau P. 1945. The yucca plant, Yucca filamentosa, and the yucca moth, Tegeticula (Pronuba) yuccasella Riley: an ecological-behavior study. Annals of the Missouri Botanical Garden 32: 373-394

Riley CV. 1892. The yucca moth and Yucca pollination. Third Annual Report Missouri Botanical Garden 99-158.

Trelease W. 1893. Further studies of yuccas and their pollination. Fourth Annual Report Missouri Botanical Garden 181-226.

MIGUEL ANGEL VILLAVICENCIO Y BLANCA ESTELA PÉREZ-ESCANDÓN. Centro de Investigaciones Biológicas. Universidad Autónoma del Estado de Hidalgo. Carr. Pachuca-Tulancingo s/n. CP. 42074 Pachuca, Hidalgo. 